EGU2020-5823

https://doi.org/10.5194/egusphere-egu2020-5823

EGU General Assembly 2020

(c) Author(s) 2021. This work is distributed under

the Creative Commons Attribution 4.0 License.

\title{
Particle Acceleration in Earth's Global Magnetosphere: a Multiple Step Process
}

\author{
Robert L. Richard ${ }^{1}$, David Schriver ${ }^{1}$, Jean Berchem ${ }^{1}$, Mostafa El-Alaoui ${ }^{1}$, Giovanni Lapenta ${ }^{2}$, and \\ Raymond J. Walker ${ }^{3}$ \\ ${ }^{1}$ UCLA, Physics and Astronomy, Los Angeles, California, USA \\ ${ }^{2}$ Centre for Plasma Astrophysics, Department of Mathematics, KU, Leuven, Belgium \\ ${ }^{3}$ UCLA, Department of Earth, Planetary, and Space Sciences, Los Angeles, California, USA
}

Particle velocity distribution functions measured by spacecraft show that suprathermal ion and electron populations are a common feature of Earth's magnetosphere. An outstanding question has been to determine the acceleration processes that lead to the formation of these suprathermal particle populations. Very often, it has been challenging to explain the high levels of energy reached by these particles by simply invoking local processes such as magnetic reconnection. In this presentation, we investigate the hypothesis that suprathermal particle populations increase if the acceleration occurs over multiple steps through different acceleration mechanisms at different spatial locations in Earth's magnetosphere. For example, particles transported to the magnetotail which have been accelerated first in the dayside reconnection region could be further accelerated in the tail reconnection regions and then gain additional energy through Fermi and/or betatron acceleration as they convected back to the dayside magnetopause. Since local kinetic processes dominate the acceleration of ions and electrons in the magnetosphere, it has been difficult to validate that hypothesis. Multiple reconnection sites and different possible acceleration regions are too distant to be included in a single kinetic simulation and global hybrid simulations cannot describe electron acceleration. To address this research problem we leverage our simulation capabilities by combining three different simulation techniques: global magnetohydrodynamic (MHD) simulations, large-scale kinetic (LSK) particle tracing simulations, and large-scale particle in cell (PIC) simulations. First, we carry out an MHD simulation driven by upstream solar wind and interplanetary magnetic field conditions for a specific time interval featuring active magnetospheric reconnection. Then we use an implicit PIC simulation of dayside reconnection with initial and boundary conditions from the MHD simulation. Next, we follow suprathermal particles from the PIC simulation globally through the MHD fields using LSK to assess their transport into the magnetotail. A final step is to perform a PIC simulation embedded in the MHD simulation of magnetotail process including the suprathermal particles arriving from the dayside as determined from the LSK simulation. Preliminary results indicate that particles energized by dayside reconnection are more likely to reach the magnetotail reconnection region. In addition, the development of enhanced high-energy tails in the particle distributions is promoted by previous energization steps during particle transport to the magnetotail reconnection region. 
